\title{
Influence of Substrate Electronic Properties on the Integrity and Functionality of an Adsorbed Fe(II) Spin-Crossover Compound
}

\author{
Sebastian Rohlf, ${ }^{\dagger, \ddagger}$ Jan Grunwald, ${ }^{\Uparrow}$ Torben Jasper-Toennies, ${ }^{\dagger}$ Sven Johannsen, ${ }^{\dagger}$ \\ Florian Diekmann, ${ }^{\dagger,}$ Michał Studniarek, ${ }^{\S}$ Richard Berndt, ${ }^{\dagger}$ Felix Tuczek, \\ Kai Rossnagel, ${ }^{\dagger, \dagger}, \|$ and Manuel Gruber, ${ }^{*} \dagger$ \\ $\dagger$ Institut für Experimentelle und Angewandte Physik, Christian-Albrechts-Universität zu \\ Kiel, 24098 Kiel, Germany \\ $\ddagger$ Ruprecht-Haensel-Labor, Christian-Albrechts-Universität zu Kiel und Deutsches \\ Elektronen-Synchrotron DESY, 24098 Kiel und 22607 Hamburg, Germany \\ \Institut für Anorganische Chemie, Christian-Albrechts-Universität zu Kiel, 24098 Kiel, \\ Germany \\ $\S$ Swiss Light Source, Paul Scherrer Institut (PSI), 5232 Villigen, Switzerland \\ \|Deutsches Elektronen-Synchrotron DESY, 22607 Hamburg, Germany \\ E-mail: gruber@physik.uni-kiel.de
}

\begin{abstract}
The bistability of spin-crossover complexes on surfaces is of great interest for potential applications. Using x-ray absorption spectroscopy, we investigated the properties of $\left[\mathrm{Fe}\left(\operatorname{pypyr}\left(\mathrm{CF}_{3}\right)_{2}\right)_{2}\right.$ (phen)] (pypyr $=2$ (2'-pyridyl)pyrrolide, phen $=1,10$-phenanthroline), a vacuum-evaporable $\mathrm{Fe}(\mathrm{II})$ complex, in direct contact to a set of substrates. The electronic properties of these substrates range from metallic to semiconducting. While dissociation is observed on metal surfaces, efficient light-induced switching is realized on semimetallic and semiconducting surfaces. This indicates that the density of states of the substrate at the Fermi level plays a role for the integrity and functionality of the adsorbed compound. In an intermediate case, namely $\left[\mathrm{Fe}\left(\operatorname{pypyr}\left(\mathrm{CF}_{3}\right)_{2}\right)_{2}\right.$ (phen)] on graphene/Ni(111), functional and dissociated species are found to coexist. This result indicates that some previous studies may deserve to be reconsidered because the possibility of coexisting intact and fragmented spin-crossover complexes was neglected.
\end{abstract}

\section{TOC Graphic}

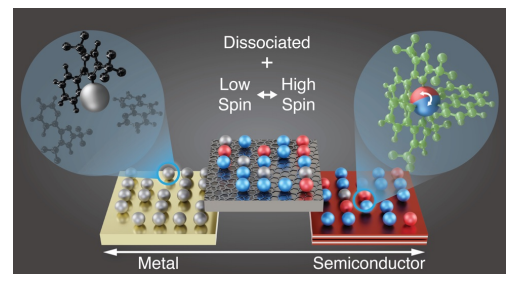

This document is the Accepted Manuscript version of a Published Work that appeared in final form in The Journal of Physical Chemistry C, copyright (C) American Chemical Society after peer review and technical editing by the publisher. To access the final edited and published work see https://www.doi.org/10.1021/acs.jpcc.9b02845. 


\section{Introduction}

The conformation and spin-state bistability of spincrossover (SCO) compounds make them attractive for applications ${ }^{1-6}$. In that respect, considerable efforts were made to realize thin films of SCO complexes using physical vapor deposition ${ }^{2-4,7-24}$. These films usually exhibit decent SCO properties. However, the direct contact to a substrate may change the properties of the SCO complexes. The interaction with metal surfaces can lead to complexes trapped in the high-spin (HS) and/or in low-spin (LS) state ${ }^{12,13,17,25-27}$ or to the fragmentation of the SCO complexes despite their integrity in the gas phase ${ }^{28-31}$. In contrast, absence of spin-state locking and efficient spin switching was evidenced for SCO complexes on highly ordered pyrolytic graphite (HOPG ${ }^{24,32-35}$ or for SCO molecules decoupled from the metal surface by a thin insulating layer ${ }^{10,25,36}$. Considering all these possible outcomes, it appears essential to determine the influence of the substrate properties on the integrity and SCO behavior of the adsorbed SCO complexes, ideally for a single SCO compound.

Here, using x-ray absorption spectroscopy (XAS) spectroscopy, we investigate sub-monolayer coverages of $\left[\mathrm{Fe}\left(\operatorname{pypyr}\left(\mathrm{CF}_{3}\right)_{2}\right)_{2}\right.$ (phen) $]$ (pypyr $=2-(2$ 'pyridyl)pyrrolide, phen = 1,10-phenanthroline) $(\mathrm{Fe}-$ pypyr) on $\mathrm{Co} / \mathrm{Cu}(100), \mathrm{Au}(111)$, graphene/Ni(111), $\mathrm{HOPG}, \mathrm{WSe}_{2}$ and $\mathrm{HfS}_{2}$. The electronic properties of these substrates range from metallic to semiconducting. While the complexes are found dissociated on metals, (light-induced) spin transitions are observed on semiconducting substrates. An intermediate scenario is found for Fe-pypyr on graphene/Ni(111), where dissociated molecules coexist with fully functional complexes. We demonstrate that this case can easily be misinterpreted as a coexistence of functional and spin-state locked molecules. Furthermore, we show that erroneous conclusions may be drawn if the quality of the sample is not reliably determined by XAS at the Fe $L_{2,3}$ edges.

\section{Experimental Methods}

\section{Sample Preparation}

Clean and flat $\mathrm{Au}(111), \mathrm{Ni}(111)$ and $\mathrm{Cu}(100)$ surfaces were prepared by several cycles of Ar sputtering (1.2 $-2 \mathrm{keV})$ and subsequent annealing $\left(500-550^{\circ} \mathrm{C}\right)$. For the preparation of graphene/ $\mathrm{Ni}(111)$, a similar procedure as in Ref. 37 was used. After the last Ar sputtering cycle, the $\mathrm{Ni}(111)$ crystal was annealed to $650^{\circ} \mathrm{C}$ for $30 \mathrm{~s}$. The temperature was then decreased to $570^{\circ} \mathrm{C}$. After $5 \mathrm{~min}$, a partial pressure of $1 \times 10^{-6} \mathrm{mbar}$ of ethylene gas was introduced in the chamber for $7 \mathrm{~min}$. For the $\mathrm{Co} / \mathrm{Cu}(100)$ samples, $6 \mathrm{ML}$ Co were deposited by electron bombardment of a $\mathrm{Co}$ rod on $\mathrm{Cu}(100)$ held at room temperature. $2 H-\mathrm{WSe}_{2}$ and $1 T-\mathrm{HfS}_{2}$ are composed of covalently bonded chalcogen-metal-chalcogen sandwich layers with weak van-der-Waals like forces between these triple layers. Atomically flat and chemi- cally saturated surfaces were obtained by scotch-tape cleaving the crystals along the basal plane at a pressure below $10^{-7}$ mbar. The HOPG crystal was cleaved ex situ followed by annealing under UHV conditions to $600{ }^{\circ} \mathrm{C}$ for one hour ${ }^{38}$. Fe-pypyr was synthesized as described in Ref. 20. The Fe-pypyr complexes were sublimed in ultrahigh vacuum (base pressure of $10^{-9} \mathrm{mbar}$ ) from a Knudsen cell heated to $170^{\circ} \mathrm{C}$ onto samples held at room temperature. The determination of the film thickness is described in the Supporting Information ((SI) section 1).

\section{X-ray Absorption Spectroscopy}

The XAS measurements were performed at the X-Treme beamline ${ }^{39}$ at the Swiss Light Source (Paul Scherrer Institute, Switzerland) in total electron yield (TEY) mode. The TEY was normalized to the photocurrent of an upstream gold mesh. A defocused beam with a photon flux estimate of $3 \times 10^{10}$ photons/s (upstream of the gold mesh) and a spot size of $0.3 \times 1.3 \mathrm{~mm}^{2}$ at the front end was employed. With these parameters and constant beamline conditions, we did not observe any evolution of the XA spectra during hours of measurements on the same sample spot, indicating the absence of photo-induced fragmentation of the Fe-pypyr complex. All XA spectra were recorded at an angle of $60^{\circ}$ between the sample normal and the circularly polarized x-rays of both helicities. All spectra, if not otherwise stated, were acquired with a magnetic field of $50 \mathrm{mT}$ in the direction of the beam to increase the signal-to-noise ratio. The spectra are averaged over 2 to 16 single measurements, low-pass filtered and polynomial backgrounds have been removed. Examples of raw data are shown in the SI (section 2). For the LIESST experiments, the spots of five $532 \mathrm{~nm}, 1 \mathrm{~mW}$ lasers were brought to coincidence on the sample.

\section{Results and Discussion}

Two electronic configurations of the central $\mathrm{Fe}^{2+}$ cation can be realized for SCO complexes, depending on the strength of the octahedral ligand field (Fig. 1a). For a strong ligand field, the six $d$ electrons of the Fe center occupy the $t_{2 g}$ set of orbitals leading to a total spin $S=0$. This state is referred to as the low-spin (LS) state. In contrast, in the high-spin (HS) state, the ligand field is weaker such that both $e_{g}$ and $t_{2 g}$ orbitals are partially filled, combining to a total spin $S=2$ (Fig. 1a). For the present study, Fe-pypyr (Fig. 1b) is employed. As shown previously ${ }^{20}$, this compound is suitable for physical vapor deposition. Thin films of this complex on $1 T-\mathrm{TiTe}_{2}$ undergo light-induced spinstate trapping (LIESST) and soft x-ray-induced excited spin-state trapping (SOXIESST) for temperatures below $100 \mathrm{~K}$ (Ref. 20). Because the spin-transition temperature of Fe-pypyr is relatively high $\left(T_{1 / 2}=390 \mathrm{~K}\right.$ for powder samples), mostly LS-to-HS transitions induced 
by $532 \mathrm{~nm}$ laser light for temperatures below $100 \mathrm{~K}$ are considered throughout the manuscript. (a)

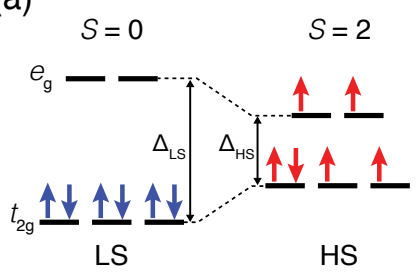

(b)

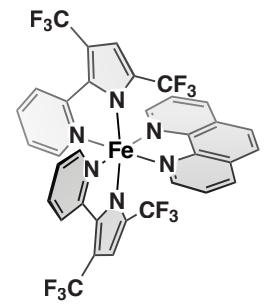

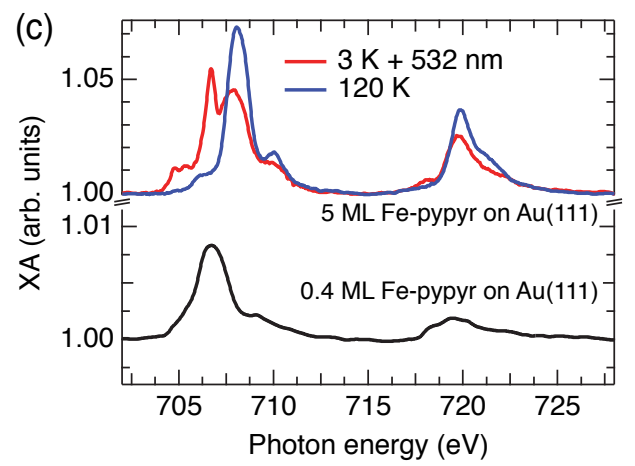

Figure 1: (a) Simplified electronic configuration of the $\mathrm{Fe}^{2+}$ cation of Fe-pypyr. Two configurations leading to two spin states may be realized depending on the strength of the ligand field. (b) Molecular model of Fe-pypyr. (c) XA spectra acquired on $5 \mathrm{ML}$ Fe-pypyr on $\mathrm{Au}(111)$ at $3 \mathrm{~K}$ under illumination with a $532 \mathrm{~nm}$ laser (red curve) and at $120 \mathrm{~K}$ (blue curve). These are used as HS and LS reference spectra, respectively. The lower spectrum (black curve) was measured on $0.4 \mathrm{ML}$ Fe-pypyr on $\mathrm{Au}(111)$ at $100 \mathrm{~K}$.

$\mathrm{XAS}$ at the Fe $L_{3,2}$ absorption edges is particularly suitable to probe the spin state averaged over an ensemble of molecules $12,16,17,19,20,22-25,30,32-34,40-44$. Indeed, the fine structure and energy of the Fe $L_{3,2}$ edges sensitively depend on the occupation and the ligand field of the Fe $d$ orbitals. This leads to characteristic and distinct $\mathrm{x}$-ray absorption (XA) spectra for the HS and LS states. The fraction of HS molecules in a given sample can be extracted from the corresponding XA spectrum at the Fe $L_{3,2}$ edges using a linear combination of the HS and LS reference spectra (SI section 3 ) as previously reported $12,16,17,19,20,22-25,30,32-34,42,44$.

Figure 1c shows XA spectra measured at $3 \mathrm{~K}$ under illumination and at $120 \mathrm{~K}$ on $5 \mathrm{ML}$ Fe-pypyr on $\mathrm{Au}(111)$. These spectra exhibit the characteristic features expected for HS and LS Fe(II), respectively ${ }^{20}$. In the following, these spectra are used as references for the HS and LS states, and the HS fractions are determined on the basis of these reference spectra. Thereby, it is assumed (as usual; see, e.g., Ref. 33) that the spin transition from LS to HS is complete in the HS reference. Reducing the film thickness to $0.4 \mathrm{ML}$ to reach the sub-ML regime leads to considerable changes (black curve in Fig. 1c). The XA spectrum has a broad $L_{3}$ edge centered at $706.7 \mathrm{eV}$. It is neither similar to the HS nor to the LS reference spectrum. No variation in the XA spectra is observed as the temperature is varied (SI section 4), which is indicative of the absence of SCO behavior. Furthermore, the spectrum is similar to that of the $\left[\mathrm{Fe}\left(\mathrm{H}_{2} \mathrm{~B}(\mathrm{pz})_{2}\right)_{2}\right]$ complex, obtained upon dissociation of the $\left[\mathrm{Fe}\left(\mathrm{H}_{2} \mathrm{~B}(\mathrm{pz})_{2}\right)_{2}\right.$ phen] compound on $\mathrm{Au}(111)$ (Ref. 28). Moreover, low-temperature scanning tunneling microscopy measurements of Fe-pypyr deposited on $\mathrm{Au}(111)$ reveal phenanthroline ligands on the surface along with fragments compatible with the formula $\left[\mathrm{Fe}\left(\operatorname{pypyr}\left(\mathrm{CF}_{3}\right)_{2}\right)\right]^{+}$(SI section 5$)$. Therefore, as will be further justified below, the black spectrum in Fig. 1c is representative of that of fragmented Fe-pypyr molecules.

Considering that the molecules withstand the sublimation process, as evident from the measurements on thicker films, the fragmentation process is most probably caused by the interaction between the molecules and the substrate. A similar behavior has been observed for other SCO compounds on $\mathrm{Au}(111)^{28,30,31}$ with exceptions ${ }^{27,45}$. Resorting to less metallic surfaces, on the other hand, has shown to promote the integrity of the molecules along with improved switching properties relative to metallic substrates ${ }^{25,30,33,36}$. We employed graphene on $\mathrm{Ni}(111)$ as a substrate because it can combine two aspects. First, a graphene layer can electronically decouple ${ }^{46}$ the Fe-pypyr molecules from the metallic Ni substrate. Second, the ferromagnetism of the $\mathrm{Ni}(111)$ layer can potentially magnetically couple to the moment of the HS Fe-pypyr molecules as observed for different systems ${ }^{47-52}$, which may be of relevance for molecular spintronics.

Fig. 2a shows XA spectra acquired on 0.4 ML Fepypyr on graphene/Ni(111) at different temperatures. Significant variations of the $L_{3,2}$ edges are observed as the temperature is changed, indicating SCO behavior. Assuming that all molecules are intact, the fraction of molecules in the HS state evolves between 50 and $100 \%$ as the temperature is varied (gray triangles in Fig. 2b). This would imply that $50 \%$ of the molecules are trapped in the HS state.

While locked spin states in sub-ML coverages have been demonstrated by STM measurements ${ }^{17,26,27}$, an alternative interpretation of the XAS data presented here is possible and actually favored. A better agreement to the XAS data is found when a fraction of the molecules $(30 \%)$ is considered as dissociated. Fig. 2c shows XA spectra acquired on 0.4 ML Fepypyr/graphene/ $\mathrm{Ni}(111)$ at $10 \mathrm{~K}$ under illumination and at $150 \mathrm{~K}$ (green curves). Although the XA spectra can be reasonably well reproduced by a superposition of LS and HS reference spectra (red curves), there are notable deviations, especially at $706.7 \mathrm{eV}$. In contrast, much less deviation is observed (solid black curves) when a fraction of molecules are considered dissociated. Actually, as significantly better fits are obtained at any temperature when a constant fraction of dissociated molecules is considered (SI section 6), we 
conclude that a fraction of complexes are fragmented on graphene/Ni(111). The corrected HS fraction as a function of temperature, evaluated by excluding the contribution from fragmented molecules, ranges from $0 \%$ to $75 \%$ (dark symbols in Fig. 2b). In other words, when taking into account that a fraction of molecules is dissociated, no complex with a locked spin state has to be postulated.

As will be shown below, the evolution of the corrected HS fraction, i.e., the HS fraction of the functional molecules, on graphene/Ni(111) matches that obtained on other substrates for sub-ML coverages of Fe-pypyr. This independently lends support to the claim that some Fe-pypyr molecules are fragmented on graphene/Ni(111). Further independent evidence of dissociation comes from the XMCD data. Indeed, small variations are observed in the fine structure of the XMCD spectra acquired on 0.4 ML Fepypyr/graphene/Ni(111) (bottom left gray spectrum in Fig. 2d) compared to that of $5 \mathrm{ML}$ Fe-pypyr $/ \mathrm{Au}(111)$ (dashed black curve in Fig. 2d) at $10 \mathrm{~K}$ under a magnetic field of $6.5 \mathrm{~T}$ (Fig. 2d). This suggests that the XMCD spectra measured on 0.4 ML Fepypyr/graphene/ $\mathrm{Ni}(111)$ do not solely originate from HS Fe-pypyr but most probably contain contributions from (magnetic) fragments. We note that LS Fe-pypyr molecules, owing to their zero magnetic moment $(S=$ 0 ), do not contribute to the XMCD signal.

Considering all the points above, we estimate that approximately $30 \%$ of the Fe-pypyr molecules are dissociated on graphene/ $\mathrm{Ni}(111)$. As it remains uncertain whether the dissociated molecules are in direct contact to the $\mathrm{Ni}(111)$ surface or reside on the graphene layer (presumably at defects), further experiments were performed. Magnetic ions in the vicinity of ferromagnetic layers usually magnetically couple to the substrate $^{29,48,49,51,52}$, such that the XMCD spectra remain non-zero as the magnetic field is reduced to approximately $0 \mathrm{~T}$. This is for instance the case for (fragmented) Fe-pypyr molecules on $\mathrm{Co} / \mathrm{Cu}(100)$ as shown in the SI (section 7). However, the XMCD signal of $0.4 \mathrm{ML}$ Fepypyr on graphene/ $\mathrm{Ni}(111)$ is essentially zero when the magnetic field is lowered to $0.1 \mathrm{~T}$ (bottom right gray spectrum in Fig. 2d). Therefore, the dissociated Fe complexes are magnetically decoupled from the $\mathrm{Ni}(111)$ substrate and most probably lie on the graphene layer. We speculate that the fragmentation of Fe-pypyr molecules is induced by defectd ${ }^{53}$ in the graphene layer with increased reactivity. For completeness, we note that the XA spectra of fragmented molecules on different substrates are similar (SI section 8) and can be modeled by multiplet calculations (SI section 5).

So far, the possibility of coexisting intact and fragmented SCO complexes on surfaces has been neglected. In turn, the distinction between complexes trapped in a given spin state and dissociated molecules is not always evident (Fig. 2c), especially when the signal-to-noise ratio of the data is low. Therefore, previous investigations, solely based on XAS data, reporting spin-state
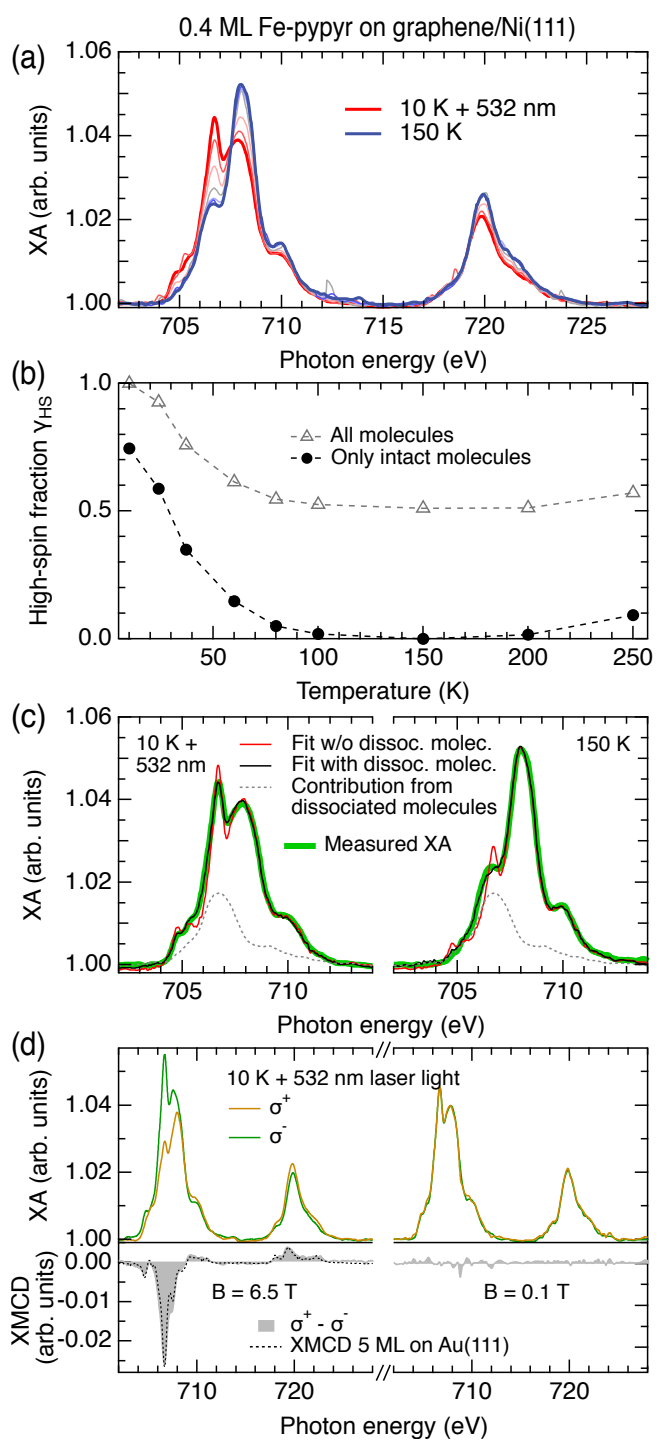

Figure 2: XA data measured on 0.4 ML Fepypyr/graphene/Ni(111). (a) XA spectra at $10 \mathrm{~K}$ under illumination (red) and $150 \mathrm{~K}$ (blue) along with XA spectra measured at intermediate temperatures under illumination with $532 \mathrm{~nm}$ laser light. (b) Fraction of HS molecules as a function of temperature evaluated considering all molecules (triangles) or only intact molecules (disks), i.e., evaluated with Eqs. 4 and 5 of the SI, respectively. The uncertainties in the HS fraction inferred from the fits are below $0.5 \%$. (c) Comparison of fits to the XA spectra acquired at $10 \mathrm{~K}$ under illumination (left) and at $150 \mathrm{~K}$ (right). While the red curves are bare linear combinations of HS and LS reference spectra, the black curves further include contributions from the dissociated molecules (SI section 6). The dashed black lines show the contribution of fragmented molecules to the spectra. (d) XMCD measurements performed at $6.5 \mathrm{~T}$ (left) and at $0.1 \mathrm{~T}$ (right). The orange and green curves are XA spectra acquired with different photon helicities. Their difference leads to the XMCD spectra (gray). For comparison, the XMCD spectrum obtained on $5 \mathrm{ML} \mathrm{Au}(111)$ is overlaid (dashed black curve). 
trapping may deserve reinterpretation. Furthermore, the purity of the compounds and the preparation conditions, which XAS at the Fe edges is not sensitive to, are of importance for the interpretation of the data. For instance, we show in the SI (section 9) that a different behavior is observed for 0.2 ML Fe-pypyr on $\mathrm{Au}(111)$, prepared with insufficient degassing of the crucible. Assuming that all the molecules are intact, the data would suggest that a large fraction $(50 \%)$ of the molecules is trapped in the HS state while the other molecules exhibit SCO behavior, in apparent contradiction to the XA spectrum (black curve) shown in Fig. 1c. However, a careful analysis of the spectra (SI section 9) leads to a different interpretation: a fraction of the molecules is in direct contact to $\mathrm{Au}(111)$ and dissociated. The remaining molecules are adsorbed on a contaminant layer on $\mathrm{Au}(111)$ and are therefore decoupled from the substrate, in turn leading to SCO. In the light of these observations, it appears challenging to prove SCO of molecules in direct contact to metal surfaces with XAS measurements alone when a fraction of the molecules are in a locked spin state and/or fragmented.

Next we employed HOPG as a substrate. Bernien et al. demonstrated the complete transition of SCO molecules in direct contact with $\mathrm{HOPG}^{33}$. To the best of our knowledge, HOPG is the only substrate reported so far where complete $\mathrm{SCO}$ is observed for sub-ML coverages of molecules. Fig. 3 shows the evolution of the HS fraction for $0.4 \mathrm{ML}$ Fe-pypyr on HOPG from 0 to $95 \%$. We speculate that the fraction of switched molecules at low temperatures may reach $100 \%$ at increased $532 \mathrm{~nm}$ laser-light irradiance. The fraction of dissociated molecules is estimated to $2 \%$, much less than the $30 \%$ found for Fe-pypyr on graphene/Ni(111). These measurements indicate that HOPG preserves the integrity and functionality of SCO complexes in direct contact to the substrate, in agreement with previous studies ${ }^{24,32-34}$. In particular, the SCO properties of Fe-pypyr are better preserved on HOPG than on graphene/ $\mathrm{Ni}(111)$ or $\mathrm{Au}(111)$.

Ossinger et al. mentioned that reducing the density of states at the Fermi level of the substrate could reduce the van-der-Waals interaction between the SCO complex and the surface and thus prevent the decomposition of the adsorbed complex ${ }^{30}$. This suggestion was tested for $\left[\mathrm{Fe}\left(\mathrm{H}_{2} \mathrm{~B}(\mathrm{pz})_{2}\right)_{2}\right.$ (phen)] (phen = 1,10-phenanthroline) deposited on metallic $\mathrm{Au}(111)$ and semimetallic $\mathrm{Bi}(111)$ surfaces. While only fragments of the $\left[\mathrm{Fe}\left(\mathrm{H}_{2} \mathrm{~B}(\mathrm{pz})_{2}\right)_{2}\right.$ (phen)] complex were found on the metal, a partial SCO transition was observed on the semimetal ${ }^{30}$. Here, a similar behavior was monitored for Fe-pypyr on $\mathrm{Au}(111)$ (metallic) and HOPG (semimetallic). In the following, we employ $\mathrm{WSe}_{2}$ and $\mathrm{HfS}_{2}$ as substrates to further decrease the density of states at the Fermi level. These are semiconducting layered transition-metal dichalcogenide materials with indirect band gaps of $1.2 \mathrm{eV}$ and $2.85 \mathrm{eV}$, respectively ${ }^{54,55}$.

The fraction of HS molecules as a function of temperature of $0.4 \mathrm{ML}$ Fe-pypyr on $\mathrm{WSe}_{2}$ and $\mathrm{HfS}_{2}$ evolves

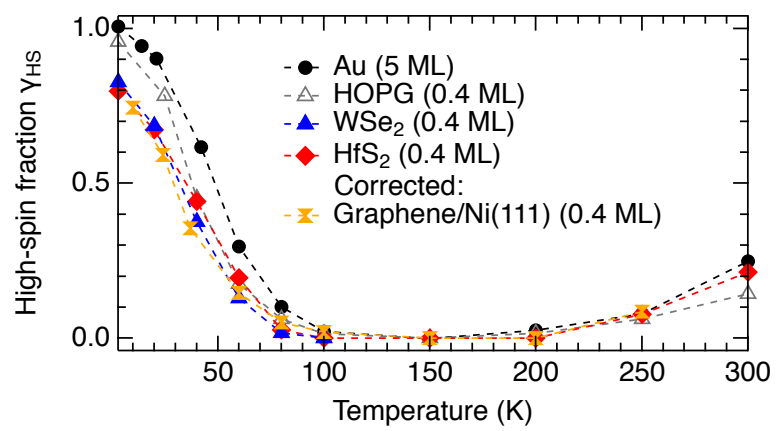

Figure 3: Evolution of the fraction of HS molecules as a function of temperature for 0.4 ML Fe-pypyr on different substrates and $5 \mathrm{ML}$ Fe-pypyr on $\mathrm{Au}(111)$ as a reference. The samples were constantly illuminated with $532 \mathrm{~nm}$ laser light. Some of the corresponding XA spectra are shown in the SI (section 3 ). Note that contributions from the dissociated molecules have been removed, i.e., the HS fraction is evaluated using Eq. 5 (SI section 6). The uncertainties in the HS fraction inferred from the fits are below $0.6 \%$.

from 0 to $80 \%$ (blue and red symbols in Fig. 3). Note that approximately $5 \%$ of the Fe-pypyr complexes were found dissociated on these substrates. A quantitative comparison between the different curves in Fig. 3 is not possible, since illumination was performed with several $532 \mathrm{~nm}$ lasers, which systematically required a readjustment after sample transfer. The irradiance most probably slightly varies from sample to sample, which in turn may influence the magnitude of the LIESST effect. Thus, the differences observed in the HS fraction of Fe-pypyr on the different substrates (Fig. 3) may either originate from different irradiances or from a (limited) influence of the surface on the LIESST process. Nonetheless, Fig. 3 shows that, within the experimental uncertainties, similar SCO performances are observed for Fe-pypyr on $\mathrm{HOPG}, \mathrm{WSe}_{2}$ and $\mathrm{HfS}_{2}$. Therefore, this study reveals that $\mathrm{WSe}_{2}$ and $\mathrm{HfS}_{2}$ are adequate substrates for efficient $\mathrm{SCO}$, i.e., spin transition without spin-state coexistence, similar to HOPG and $\mathrm{Cu}_{2} \mathrm{~N}$ substrates.

\section{Conclusion}

In conclusion, we investigated sub-ML coverages of Fepypyr on different substrates with distinct electronic properties. Efficient light-induced spin switching is observed on semimetallic HOPG and semiconducting substrates $\left(\mathrm{WSe}_{2}\right.$ and $\mathrm{HfS}_{2}$ ). However, Fe-pyrpyr dissociates in direct contact to metallic substrates $(\mathrm{Au}(111)$ and $\mathrm{Co} / \mathrm{Cu}(100))$. This agrees with a previous suggestion $^{30}$ that reducing the density of states of the substrate at the Fermi level is favorable for the integrity and functionality of deposited SCO compounds. Furthermore, a thorough analysis of an intermediate 
case (Fe-pypyr on graphene/Ni(111)) shows that dissociated molecules may easily be confused with complexes trapped in a given spin state. The experimental distinction between the two possibilities is of significant importance for accurate estimation of the robustness and SCO efficiency of complexes on surfaces, which in turn are ingredients for the design of new robust spincrossover complexes.

Acknowledgement We thank W. Kuch for helpful discussions about the estimation of adsorbate thickness using XAS. We acknowledge funding from the Deutsche Forschungsgemeinschaft (DFG) via Sonderforschungsbereich 677 . We thank all members of the XTreme beam line at Swiss Light Source for assistance in using the end station. M. S. acknowledges funding from the European Union's Horizon 2020 research and innovation programme under the Marie Sklodowska-Curie grant agreement No 701647 and Swiss National Science Foundation (Grant No. 200021_165774/1).

Supporting Information Available: Additional information is available about molecular coverage estimation, examples of raw data, determination of the HS fraction, additional spectra of Fe-pypyr fragments on $\mathrm{Au}(111)$, identification of the Fe-pypyr fragments on $\mathrm{Au}(111)$ with low temperature scanning tunneling microscopy and multiplet calculations, distinction between coexistence of dissociated and functional molecules and spin-state coexistence, data of Fe-pypyr on $\mathrm{Co} / \mathrm{Cu}(100)$, comparison of dissociated molecules on different substrates and peculiar results of $0.2 \mathrm{ML}$ Fepypyr on $\mathrm{Au}(111)$ prepared under inappropriate conditions. This material is available free of charge via the Internet at http://pubs.acs.org/.

\section{References}

(1) Halcrow, M. A. Spin-Crossover Materials: Properties and Applications, 1st ed.; Wiley: Chichester, West Sussex, United Kingdom, 2013.

(2) Shepherd, H. J.; Molnár, G.; Nicolazzi, W.; Salmon, L.; Bousseksou, A. Spin Crossover at the Nanometre Scale. Eur. J. Inorg. Chem. 2012, 2013, 653-661.

(3) Molnár, G.; Salmon, L.; Nicolazzi, W.; Terki, F.; Bousseksou, A. Emerging Properties and Applications of Spin Crossover Nanomaterials. J. Mater. Chem. C 2014, 2, 1360.

(4) Lefter, C.; Davesne, V.; Salmon, L.; Molnár, G.; Demont, P.; Rotaru, A.; Bousseksou, A. Charge Transport and Electrical Properties of Spin Crossover Materials: Towards Nanoelectronic and Spintronic Devices. Magnetochemistry 2016, 2, 18.
(5) Molnár, G.; Rat, S.; Salmon, L.; Nicolazzi, W.; Bousseksou, A. Spin Crossover Nanomaterials: From Fundamental Concepts to Devices. Adv. Mater. 2017, 17003862.

(6) Bellec, A.; Lagoute, J.; Repain, V. Molecular Electronics: Scanning Tunneling Microscopy and Single-Molecule Devices. Comptes Rendus Chimie 2018, 21, 1287-1299.

(7) Shi, S.; Schmerber, G.; Arabski, J.; Beaufrand, J.B.; Kim, D. J.; Boukari, S.; Bowen, M.; Kemp, N. T.; Viart, N.; Rogez, G. et al. Study of Molecular Spin-Crossover Complex $\mathrm{Fe}(\text { phen })_{2}(\mathrm{NCS})_{2}$ Thin Films. Appl. Phys. Lett. 2009, 95, 043303.

(8) Naggert, H.; Bannwarth, A.; Chemnitz, S.; von Hofe, T.; Quandt, E.; Tuczek, F. First Observation of Light-Induced Spin Change in Vacuum Deposited Thin Films of Iron Spin Crossover Complexes. Dalton Trans. 2011, 40, 6364-6366.

(9) Mahfoud, T.; Molnár, G.; Cobo, S.; Salmon, L.; Thibault, C.; Vieu, C.; Demont, P.; Bousseksou, A. Electrical Properties and Non-Volatile Memory Effect of the $\left[\mathrm{Fe}\left(\mathrm{HB}(\mathrm{pz})_{3}\right)_{2}\right]$ Spin Crossover Complex Integrated in a Microelectrode Device. Appl. Phys. Lett. 2011, 99, 053307.

(10) Gopakumar, T. G.; Matino, F.; Naggert, H.; Bannwarth, A.; Tuczek, F.; Berndt, R. ElectronInduced Spin Crossover of Single Molecules in a Bilayer on Gold. Angew. Chem., Int. Ed. 2012, 51, 6262-6266.

(11) Palamarciuc, T.; Oberg, J. C.; El Hallak, F.; Hirjibehedin, C. F.; Serri, M.; Heutz, S.; Létard, J. F.; Rosa, P. Spin Crossover Materials Evaporated Under Clean High Vacuum and Ultra-High Vacuum Conditions: From Thin Films to Single Molecules. J. Mater. Chem. 2012, 22, 9690-9695.

(12) Warner, B.; Oberg, J. C.; Gill, T. G.; El Hallak, F.; Hirjibehedin, C. F.; Serri, M.; Heutz, S.; Arrio, M.A.; Sainctavit, P.; Mannini, M. et al. TemperatureAnd Light-Induced Spin Crossover Observed by XRay Spectroscopy on Isolated Fe(II) Complexes on Gold. J. Phys. Chem. Lett. 2013, 4, 1546-1552.

(13) Pronschinske, A.; Chen, Y.; Lewis, G. F.; Shultz, D. A.; Calzolari, A.; Buongiorno Nardelli, M.; Dougherty, D. B. Modification of Molecular Spin Crossover in Ultrathin Films. Nano Lett. 2013, 13, 1429-1434.

(14) Pronschinske, A.; Bruce, R. C.; Lewis, G.; Chen, Y.; Calzolari, A.; Buongiorno-Nardelli, M.; Shultz, D. A.; You, W.; Dougherty, D. B. 
Iron(II) Spin Crossover Films on Au(111): Scanning Probe Microscopy and Photoelectron Spectroscopy. Chem. Commun. 2013, 49, 10446 10452 .

(15) Ludwig, E.; Naggert, H.; Kalläne, M.; Rohlf, S.; Kröger, E.; Bannwarth, A.; Quer, A.; Rossnagel, K.; Kipp, L.; Tuczek, F. Iron(II) SpinCrossover Complexes in Ultrathin Films: Electronic Structure and Spin-State Switching by Visible and Vacuum-UV Light. Angew. Chem., Int. Ed. 2014, 53, 3019-3023.

(16) Davesne, V.; Gruber, M.; Studniarek, M.; Doh, W. H.; Zafeiratos, S.; Joly, F., L. Sirotti; Silly, M. G.; Gaspar, A. B.; Real, J. A.; Schmerber, G. et al. Hysteresis and Change of Transition Temperature in Thin Films of Fe[Me $\mathrm{Myrz}_{3} \mathrm{BH}_{2}$, a New Sublimable Spin-Crossover Molecule. J. Chem. Phys. 2015, 142, 194702.

(17) Gruber, M.; Miyamachi, T.; Davesne, V.; Bowen, M.; Boukari, S.; Wulfhekel, W.; Alouani, M.; Beaurepaire, E. Spin Crossover in $\mathrm{Fe}(\text { phen })_{2}(\mathrm{NCS})_{2}$ Complexes on Metallic Surfaces. J. Chem. Phys. 2017, 146, 092312.

(18) Iasco, O.; Boillot, M.-L.; Bellec, A.; Guillot, R.; Rivière, E.; Mazerat, S.; Nowak, S.; Morineau, D.; Brosseau, A.; Miserque, F. et al. The Disentangling of Hysteretic Spin Transition, Polymorphism and Metastability in Bistable Thin Films Formed by Sublimation of bis(scorpionate) Fe(II) Molecules. J. Mater. Chem. C 2017, 5, 11067.

(19) Bairagi, K.; Bellec, A.; Fourmental, C.; Iasco, O.; Lagoute, J.; Chacon, C.; Girard, Y.; Rousset, S.; Choueikani, F.; Otero, E. et al. Temperature-, Light-, and Soft X-ray-Induced Spin Crossover in a Single Layer of $\mathrm{Fe}^{I I}$-Pyrazolylborate Molecules in Direct Contact with Gold. J. Phys. Chem. C 2017, 122, 727-731.

(20) Rohlf, S.; Gruber, M.; Flöser, B. M.; Grunwald, J.; Jarausch, S.; Diekmann, F.; Kalläne, M.; JasperToennies, T.; Buchholz, A.; Plass, W. et al. LightInduced Spin Crossover in an Fe(II) Low-Spin Complex Enabled by Surface Adsorption. J. Phys. Chem. Lett. 2018, 9, 1491-1496.

(21) Poggini, L.; Milek, M.; Londi, G.; Naim, A.; Poneti, G.; Squillantini, L.; Magnani, A.; Totti, F.; Rosa, P.; M. Khusniyarov, M. et al. Room Temperature Control of Spin States in a Thin Film of a Photochromic Iron(II) Complex. Mater. Horiz. 2018, 5, 506-513.

(22) Schleicher, F.; Studniarek, M.; Kumar, K. S.; Urbain, E.; Katcko, K.; Chen, J.; Frauhammer, T.; Hervé, M.; Halisdemir, U.; Kandpal, L. M. et al.
Linking Electronic Transport through a Spin Crossover Thin Film to the Molecular Spin State Using X-ray Absorption Spectroscopy Operando Techniques. ACS Appl. Mater. Interfaces 2018, 10, 31580-31585.

(23) Wäckerlin, C.; Donati, F.; Singha, A.; Baltic, R.; Decurtins, S.; Liu, S.-X.; Rusponi, S.; Dreiser, J. Excited Spin-State Trapping in Spin Crossover Complexes on Ferroelectric Substrates. J. Phys. Chem. C 2018, 122, 8202-8208.

(24) Kipgen, L.; Bernien, M.; Ossinger, S.; Nickel, F.; Britton, A. J.; Arruda, L. M.; Naggert, H.; Luo, C.; Lotze, C.; Ryll, H. et al. Evolution of Cooperativity in the Spin Transition of an Iron(II) Complex on a Graphite Surface. Nat. Commun. 2018, 9, 2984.

(25) Miyamachi, T.; Gruber, M.; Davesne, V.; Bowen, M.; Boukari, S.; Joly, L.; Scheurer, F.; Rogez, G.; Yamada, T. K.; Ohresser, P. et al. Robust Spin Crossover and Memristance Across a Single Molecule. Nat. Commun. 2012, 3, 938.

(26) Gruber, M.; Davesne, V.; Bowen, M.; Boukari, S.; Beaurepaire, E.; Wulfhekel, W.; Miyamachi, T. Spin State of Spin-Crossover Complexes: From Single Molecules to Ultrathin Films. Phys. Rev. $B$ 2014, 89, 195415.

(27) Bairagi, K.; Iasco, O.; Bellec, A.; Kartsev, A.; Li, D.; Lagoute, J.; Chacon, C.; Girard, Y.; Rousset, S.; Miserque, F. et al. Molecular-Scale Dynamics of Light-Induced Spin Cross-Over in a Two-Dimensional Layer. Nat. Commun. 2016, 7, 12212 .

(28) Gopakumar, T. G.; Bernien, M.; Naggert, H.; Matino, F.; Hermanns, C. F.; Bannwarth, A.; Mühlenberend, S.; Krüger, A.; Krüger, D.; Nickel, F. et al. Spin-Crossover Complex on $\mathrm{Au}(111)$ : Structural and Electronic Differences Between Mono- And Multilayers. Chem. Eur. J. 2013, 19, 15702-15709.

(29) Gueddida, S.; Gruber, M.; Miyamachi, T.; Beaurepaire, E.; Wulfhekel, W.; Alouani, M. Exchange Coupling of Spin-Crossover Molecules to Ferromagnetic Co Islands. J. Phys. Chem. Lett. 2016, 7, 900-904.

(30) Ossinger, S.; Naggert, H.; Kipgen, L.; JasperToennies, T.; Rai, A.; Rudnik, J.; Nickel, F.; Arruda, L. M.; Bernien, M.; Kuch, W. et al. VacuumEvaporable Spin-Crossover Complexes in Direct Contact With a Solid Surface: Bismuth Versus Gold. J. Phys. Chem. C 2017, 121, 1210-1219. 
(31) Knaak, T.; González, C.; Dappe, Y. J.; Harzmann, G. D.; Brandl, T.; Mayor, M.; Berndt, R.; Gruber, M. Fragmentation and Distortion of Terpyridine-Based Spin-Crossover Complexes on $\mathrm{Au}(111)$. J. Phys. Chem. C 2019,

(32) Bernien, M.; Wiedemann, D.; Hermanns, C. F.; Krüger, A.; Rolf, D.; Kroener, W.; Müller, P.; Grohmann, A.; Kuch, W. Spin Crossover in a Vacuum-Deposited Submonolayer of a Molecular Iron(II) Complex. J. Phys. Chem. Lett. 2012, 3, 3431-3434.

(33) Bernien, M.; Naggert, H.; Arruda, L. M.; Kipgen, L.; Nickel, F.; Miguel, J.; Hermanns, C. F.; Krüger, A.; Krüger, D.; Schierle, E. et al. Highly Efficient Thermal and Light-Induced Spin-State Switching of an Fe(II) Complex in Direct Contact With a Solid Surface. ACS Nano 2015, 9, 8960-8966.

(34) Kipgen, L.; Bernien, M.; Nickel, F.; Naggert, H.; Britton, A. J.; Arruda, L. M.; Schierle, E.; Weschke, E.; Tuczek, F.; Kuch, W. Soft-X-RayInduced Spin-State Switching of an Adsorbed Fe(II) Spin-Crossover Complex. J. Phys.: Condens. Matter 2017, 29, 394003.

(35) Kuch, W.; Bernien, M. Controlling the Magnetism of Adsorbed Metal-Organic Molecules. J. Phys.: Condens. Matter 2017, 29, 023001.

(36) Jasper-Toennies, T.; Gruber, M.; Karan, S.; Jacob, H.; Tuczek, F.; Berndt, R. Robust and Selective Switching of an Fe ${ }^{\mathrm{III}}$ Spin-Crossover Compound on $\mathrm{Cu}_{2} \mathrm{~N} / \mathrm{Cu}(100)$ with Memristance Behavior. Nano Lett. 2017, 17, 6613.

(37) Addou, R.; Dahal, A.; Sutter, P.; Batzill, M. Monolayer Graphene Growth on $\mathrm{Ni}(111)$ by Low Temperature Chemical Vapor Deposition. Appl. Phys. Lett. 2012, 100, 021601.

(38) Xie, W.; Weng, L.-T.; Ng, K. M.; Chan, C. K.; Chan, C.-M. Clean graphene surface through high temperature annealing. Carbon 2015, 94, 740 748 .

(39) Piamonteze, C.; Flechsig, U.; Rusponi, S.; Dreiser, J.; Heidler, J.; Schmidt, M.; Wetter, R.; Calvi, M.; Schmidt, T.; Pruchova, H. et al. XTreme Beamline at SLS: X-Ray Magnetic Circular and Linear Dichroism at High Field and Low Temperature. J. Synchrotron Radiat. 2012, 19, 661674 .

(40) Cartier dit Moulin, C.; Rudolf, P.; Flank, A. M.; Chen, C. T. Spin Transition Evidenced by Soft X-Ray Absorption Spectroscopy. J. Phys. Chem. 1992, 96, 6196-6198.
(41) Collison, D.; Garner, C. D.; McGrath, C. M.; Mosselmans, J. F. W.; Roper, M. D.; Seddon, J. M. W.; Sinn, E.; Young, N. A. Soft X-Ray Induced Excited Spin State Trapping and Soft XRay Photochemistry at the Iron $\mathrm{L}_{2,3}$ Edge in $\left[\mathrm{Fe}(\text { phen })_{2}(\mathrm{NCS})_{2}\right]$ and $\left[\mathrm{Fe}(\text { phen })_{2}(\mathrm{NCSe})_{2}\right]$ (phen = 1,10-phenanthroline). Dalton Trans. 1997, 4371-4376.

(42) Davesne, V.; Gruber, M.; Miyamachi, T.; Da Costa, V.; Boukari, S.; Scheurer, F.; Joly, L.; Ohresser, P.; Otero, E.; Choueikani, F. et al. First Glimpse of the Soft X-Ray Induced Excited Spin-State Trapping Effect Dynamics on Spin Cross-Over Molecules. J. Chem. Phys. 2013, 139, 074708.

(43) Zhang, X.; Costa, P. S.; Hooper, J.; Miller, D. P.; N'Diaye, A. T.; Beniwal, S.; Jiang, X.; Yin, Y.; Rosa, P.; Routaboul, L. et al. Locking and Unlocking the Molecular Spin Crossover Transition. Adv. Mater. 2017, 29, 1702257.

(44) Kumar, K. S.; Studniarek, M.; Heinrich, B.; Arabski, J.; Schmerber, G.; Bowen, M.; Boukari, S.; Beaurepaire, E.; Dreiser, J.; Ruben, M. Engineering On-Surface Spin Crossover: Spin-State Switching in a Self-Assembled Film of VacuumSublimable Functional Molecule. Adv. Mater. 2018, 1705416.

(45) Jasper-Tönnies, T.; Gruber, M.; Karan, S.; Jacob, H.; Tuczek, F.; Berndt, R. Deposition of a Cationic Fe $\mathrm{Fe}^{\mathrm{III}}$ Spin-Crossover Complex on $\mathrm{Au}(111)$ : Impact of the Counter Ion. J. Phys. Chem. Lett. 2017, 8, 1569-1573.

(46) Uihlein, J.; Peisert, H.; Adler, H.; Glaser, M.; Polek, M.; Ovsyannikov, R.; Chassé, T. Interface between $\mathrm{FePc}$ and $\mathrm{Ni}(111)$ : Influence of Graphene Buffer Layers. J. Phys. Chem. C 2014, 118, 10106-10112.

(47) Hermanns, C. F.; Tarafder, K.; Bernien, M.; Krüger, A.; Chang, Y.-M.; Oppeneer, P. M.; Kuch, W. Magnetic Coupling of Porphyrin Molecules Through Graphene. Adv. Mater. 2013, 25, 3473-3477.

(48) Scheybal, A.; Ramsvik, T.; Bertschinger, R.; Putero, M.; Nolting, F.; Jung, T. A. Induced Magnetic Ordering in a Molecular Monolayer. Chem. Phys. Lett. 2005, 411, 214-220.

(49) Wende, H.; Bernien, M.; Luo, J.; Sorg, C.; Ponpandian, N.; Kurde, J.; Miguel, J.; Piantek, M.; Xu, X.; Eckhold, P. et al. Substrate-Induced Magnetic Ordering and Switching of Iron Porphyrin Molecules. Nat. Mater. 2007, 6, 516-520. 
(50) Candini, A.; Bellini, V.; Klar, D.; Corradini, V.; Biagi, R.; De Renzi, V.; Kummer, K.; Brookes, N. B.; del Pennino, U.; Wende, H. et al. Ferromagnetic Exchange Coupling between $\mathrm{Fe} \mathrm{Ph}-$ thalocyanine and $\mathrm{Ni}(111)$ Surface Mediated by the Extended States of Graphene. J. Phys. Chem. C 2014, 118, 17670-17676.

(51) Gruber, M.; Ibrahim, F.; Boukari, S.; Isshiki, H.; Joly, L.; Peter, M.; Studniarek, M.; Da Costa, V.; Jabbar, H.; Davesne, V. et al. Exchange Bias and Room-Temperature Magnetic Order in Molecular Layers. Nat. Mater. 2015, 14, 981-984.

(52) Gruber, M.; Ibrahim, F.; Boukari, S.; Joly, L.; Da Costa, V.; Studniarek, M.; Peter, M.; Isshiki, H.; Jabbar, H.; Davesne, V. et al. SpinDependent Hybridization between Molecule and Metal at Room Temperature through Interlayer Exchange Coupling. Nano Lett. 2015, 15, 79217926.

(53) Jacobson, P.; Stöger, B.; Garhofer, A.; Parkinson, G. S.; Schmid, M.; Caudillo, R.; Mittendorfer, F.; Redinger, J.; Diebold, U. Disorder and Defect Healing in Graphene on Ni(111). J. Phys. Chem. Lett. 2012, 3, 136-139.

(54) Traving, M.; Boehme, M.; Kipp, L.; Skibowski, M.; Starrost, F.; Krasovskii, E. E.; Perlov, A.; Schattke, W. Electronic Structure of $\mathrm{WSe}_{2}$ : A Combined Photoemission and Inverse Photoemission Study. Phys. Rev. B 1997, 55, 10392-10399.

(55) Traving, M.; Seydel, T.; Kipp, L.; Skibowski, M.; Starrost, F.; Krasovskii, E. E.; Perlov, A.; Schattke, W. Combined Photoemission and Inverse Photoemission Study of $\mathrm{HfS}_{2}$. Phys. Rev. B 2001, 63. 


\section{Supporting Information:}

\section{Influence of Substrate Electronic Properties}

on the Integrity and Functionality of an

\section{Adsorbed Fe(II) Spin-Crossover Compound}

Sebastian Rohlf, ${ }^{\dagger}, \underset{j}{ }$ Jan Grunwald, ${ }^{\text {T Torben Jasper-Toennies, }}{ }^{\dagger}$ Sven Johannsen, ${ }^{\dagger}$ Florian Diekmann, ${ }^{\dagger,}$ Michał Studniarek, ${ }^{\S}$ Richard Berndt, ${ }^{\dagger}$ Felix Tuczek, Kai Rossnagel, ${ }^{\dagger,+, \|}$ and Manuel Gruber, ${ }^{*} \dagger$

$\dagger$ Institut für Experimentelle und Angewandte Physik, Christian-Albrechts-Universität zu Kiel, 24098 Kiel, Germany

$\ddagger$ Ruprecht-Haensel-Labor, Christian-Albrechts-Universität zu Kiel und Deutsches Elektronen-Synchrotron DESY, 24098 Kiel und 22607 Hamburg, Germany 9Institut für Anorganische Chemie, Christian-Albrechts-Universität zu Kiel, 24098 Kiel, Germany

$\S$ Swiss Light Source, Paul Scherrer Institut (PSI), 5232 Villigen, Switzerland ||Deutsches Elektronen-Synchrotron DESY, 22607 Hamburg, Germany

E-mail: gruber@physik.uni-kiel.de 


\section{S1 Molecular coverage estimation}

The coverage estimation is mostly based on the frequency shift of a quartz microbalance, which is calibrated by STM measurements. Integrated $\mathrm{Fe} L_{3,2}$ edge-intensities of the x-ray absorption (XA) spectra were used to crosscheck the coverage.

Combined scanning tunneling microscopy (STM) and XA spectroscopy measurements were performed on the same sample, without breaking the ultra-high vacuum conditions. Figure S1a shows a room-temperature constant-current STM topograph recorded upon sublimation of Fe-pypyr complexes on $\mathrm{Au}(111)$. Molecular islands are discernible but are more evident in the current channel (Figure S1b). In Figure S1b, the molecular islands occupy $40 \%$ of the surface, which we define as a coverage of 0.4 monolayer (ML). A similar analysis on Figure S1a leads to a coverage estimate of $45 \%$. In order to evaluate the potential error of the coverage estimation, the topograph shown in Figure S1b is divided in 8 topographs of equal areas. Coverage determinations independently carried out on each subtopograph lead to an average value of $\mu=0.4 \mathrm{ML}$ and a standard deviation of $\sigma=0.2 \mathrm{ML}$. We therefore consider a coverage of $(0.4 \pm 0.2) \mathrm{ML}$ for this sample. The following functions were employed:

$$
\mu=\frac{1}{8} \sum_{i=1}^{8} C_{i}, \text { and } \sigma=\sqrt{\frac{1}{8-1} \sum_{i=1}^{8}\left|C_{i}-\mu\right|^{2}}
$$

where $C_{i}$ is the coverage estimate inferred from subtopograph $i$.

A coverage of $0.4 \mathrm{ML}$ corresponds to a given frequency shift of a quartz microbalance, which we estimated from calibrations before and after the deposition of molecules. All further coverage estimates are primarily based on the frequency shift of the quartz microbalance, which is proportional to the amount of deposited molecules. As similar coverages on the different samples were desired for the present study, the integrated Fe $L_{3,2}$ edge intensity

of background-subtracted XA spectra should be constant. Indeed, the total electron yield of an adsorbate layer of thickness $\Delta x$ and x-ray absorption coefficient $\mu_{A}$ on a semi-infinite substrate with a x-ray absorption coefficient $\mu_{C}$ reads $^{1,2}$ : 

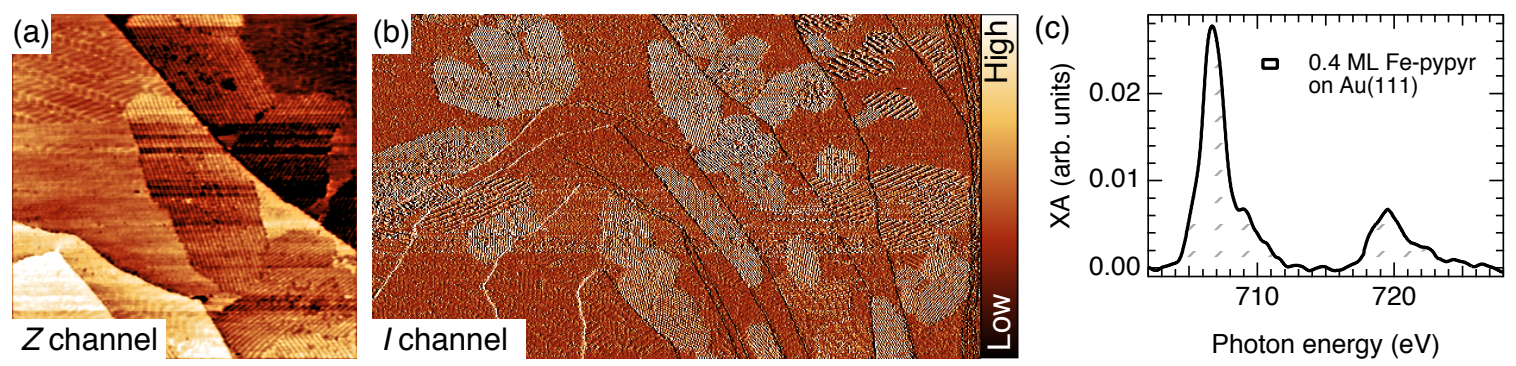

Figure S1: (a) Room-temperature constant-current STM topograph (300 nm wide) of a submonolayer coverage of Fe-pypyr on $\mathrm{Au}(111)$. (b) Overview STM topograph exhibiting molecular islands ( $V=2.5 \mathrm{~V}, I_{\text {set }}=20 \mathrm{pA}, 1 \mu \mathrm{m}$ wide). For better visibility of the islands, the deviation of the current from the set point is shown. (c) XA spectrum acquired on the same sample at $300 \mathrm{~K}$ (background subtracted).

$$
Y_{\text {sample }} \sim\left(1-e^{-\frac{\Delta x}{\lambda_{e}}}\right) \lambda_{e} \mu_{A}+e^{-\frac{\Delta x}{\lambda_{e}}} \lambda_{e} \mu_{C}
$$

where $\lambda_{e}$ is the electron escape length. The total electron yield of the Au grid is given by $Y_{\text {grid }} \sim \lambda_{e} \mu_{G}$ with $\mu_{G}$ the x-ray absorption coefficient of the grid (Ref. 2). The sample yield divided by the grid yield $Y_{\text {sample/grid }}$ is a quantity independent of the photon flux. $Y_{\text {sample/grid }}$ from different measurements should only be compared when realized on the same beamline and ideally during the same beamtime. This was the case for the present study. The edge jump of spectra solely normalized by the grid current is then given by ${ }^{2}$ :

$$
Y_{\text {sample/grid }}^{\text {edge }}-Y_{\text {sample/grid }}^{\text {pre-edge }} \sim\left(1-e^{-\frac{\Delta x}{\lambda_{e}}}\right)\left(\frac{\mu_{A}^{\text {edge }}-\mu_{A}^{\text {pre-edege }}}{\mu_{G}}\right)
$$

where $\mu_{C}$ is assumed to be constant over the energy range of interest. Equation S3 suggests that, for the same thickness of adsorbate $\Delta x$, the edge jump of XA spectra solely normalized to grid current should be constant on different substrates, provided that $\lambda_{e}$ stays constant. Different growth modes of the molecules or different magnetic fields on the sample may effectively lead to a change in $\lambda_{e}$. Furthermore, employing Equation S3 to compare thicknesses on different substrates requires the x-ray absorption coefficient of the adsorbate $\mu_{A}$ to be substrate independent. This notably implies that the number of $d$ holes in the adsorbate 
should remain constant on the different substrates, i.e., same oxidation state of the metal center for transition-metal complexes.

In the following, we consider the integral of Equation S3 over the energy range of interest. A XA spectrum was measured at the Fe $L_{3,2}$ edges on the very same sample as investigated by STM (Fig. S1c), with an integrated $L_{3,2}$ intensity of 0.097 arbitrary units (gray shaded area). For the samples of 0.4 ML Fe-pypyr on different substrates, variations of the integrated Fe $L_{3,2}$ intensity of less than $35 \%$ were ensured. This indicates that the Fe-pypyr coverage, primarily estimated with the quartz balance, is relatively constant on the different substrates. It is therefore safe to assume sub-monolayer coverages for the investigated samples.

\section{S2 Examples of raw data}

Figure S2 shows examples of averaged raw and processed data acquired on different samples at different temperatures.

The red curves in the left panels of Figure S2 are obtained by averaging 2 to 4 raw spectra and the blue curves represent the linear background associated to the spectra. Subtraction of the linear background from the averaged spectra leads to the spectra shown in red in the right panels of Figure S2. A low-pass filter is then applied to the data (black curves in Figure S2), by taking care that the eventual induced changes in the $L_{3,2}$ edges do not exceed the noise level of the data. 

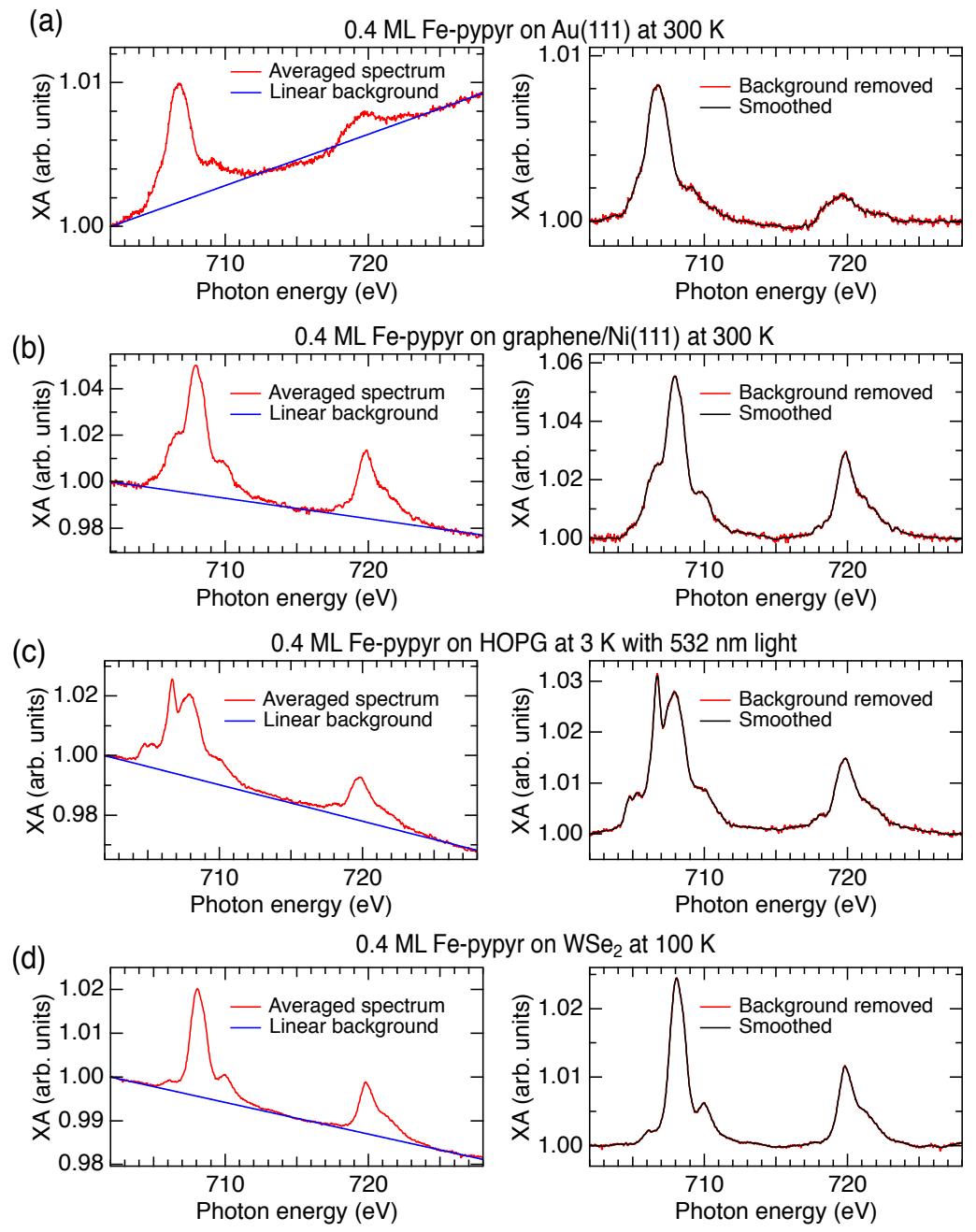

Figure S2: Examples of raw and processed spectra acquired on 0.4 ML Fe-pypyr on (a) $\mathrm{Au}(111)$ at $300 \mathrm{~K}$, (b) graphene/Ni(111) at $300 \mathrm{~K}$, (c) HOPG at $3 \mathrm{~K}$ with $532 \mathrm{~nm}$ laser illumination and (d) $\mathrm{WSe}_{2}$ at $100 \mathrm{~K}$. The red and blue curves in the left panels represent averages of 2 to 4 raw spectra and an estimate of the linear background, respectively. Background subtracted data before (red curves) and after low-pass filtering (black curves) are shown in the right panels. 


\section{S3 Determination of the high-spin fraction}
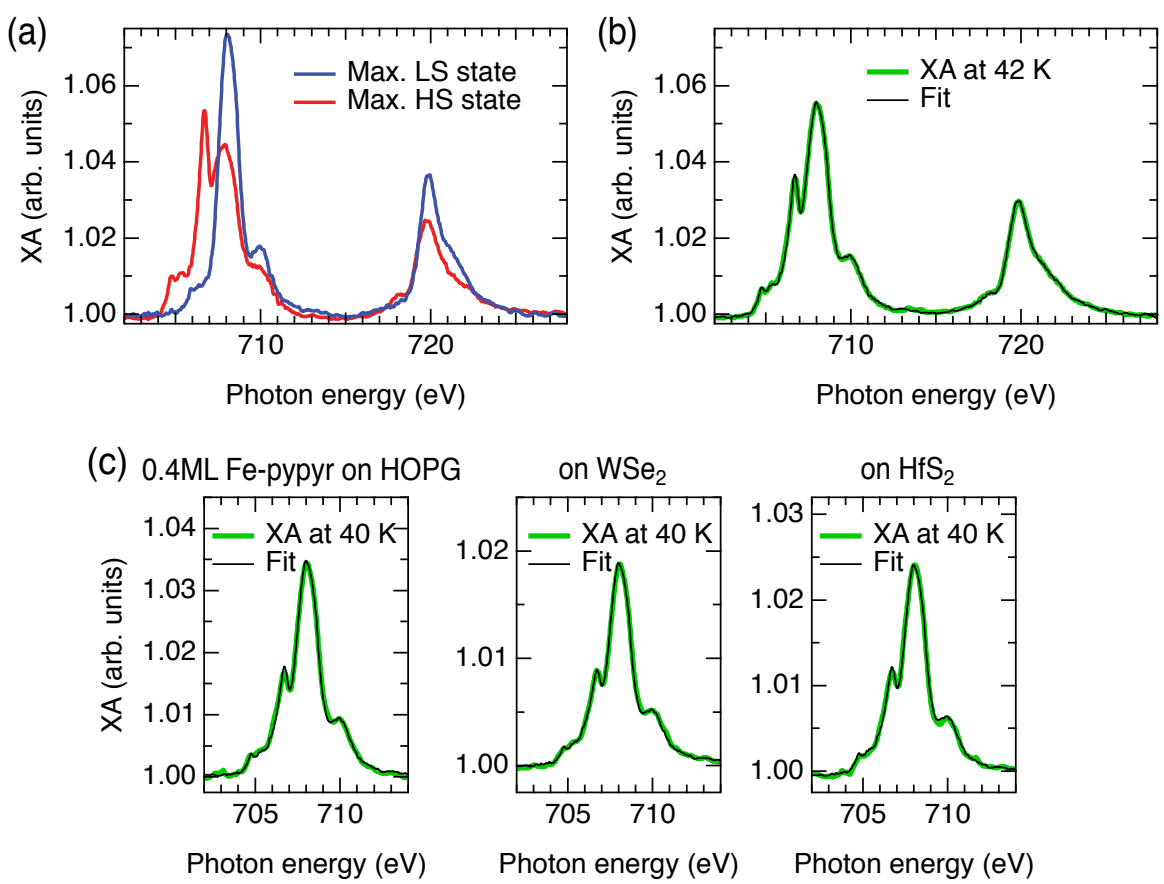

Figure S3: (a) XA spectra of 5 ML Fe-pypyr on $\mathrm{Au}(111)$ at $3 \mathrm{~K}$ under $532 \mathrm{~nm}$ laser light irradiation (red) and at $120 \mathrm{~K}$ (blue) used as reference for maximum HS and LS state, respectively. (b) XA spectrum of $5 \mathrm{ML}$ Fe-pypyr on $\mathrm{Au}(111)$ acquired at $42 \mathrm{~K}$ compared to the linear combination fit (Equation S4). (c) Exemplary comparison between fits and measured spectra of $0.4 \mathrm{ML}$ Fe-pypyr on $\mathrm{HOPG}, \mathrm{WSe}_{2}$ and $\mathrm{HfS}_{2}$.

The XA spectrum of intact Fe(II) spin-crossover complexes is a linear combination of the characteristic HS and LS spectra. As reference for the characteristic HS and LS spectra, we took the one acquired on $5 \mathrm{ML}$ Fe-pypyr/Au(111) at $3 \mathrm{~K}$ under illumination and the one at $120 \mathrm{~K}$, respectively (Fig. S3a). We note that the spin transition from LS to HS may be incomplete in the HS reference (red curve in Fig. S3a), for instance because of insufficient laser illumination. The fractions of HS molecules given in the manuscript are relative to these reference spectra. The HS fraction is determined from a fit to the XA spectrum using ${ }^{3}$ :

$$
X A S_{\mathrm{mix}}=a\left[\gamma_{\mathrm{HS}} X A S_{\mathrm{HS}}+\left(1-\gamma_{\mathrm{HS}}\right) X A S_{\mathrm{LS}}\right]
$$


where $a$ and $\gamma_{\mathrm{HS}}$ are adjustable parameters. The agreement between the fit and the XA spectra is remarkable as shown in Figures S3b-c.

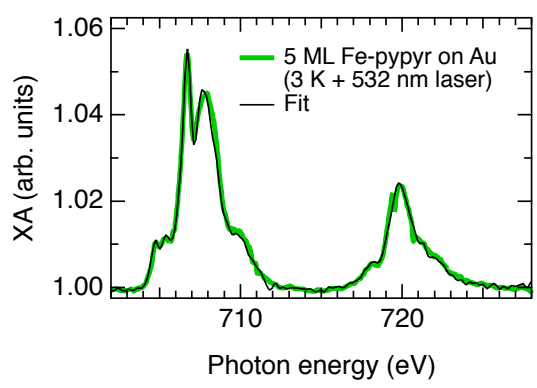

Figure S4: XA spectrum acquired on 5 ML Fe-pypyr on $\mathrm{Au}(111)$ at $3 \mathrm{~K}$ under illumination with $532 \mathrm{~nm}$ laser light (green curve) along with a fit using Equation S4 with HS and LS references from Ref. 3 (black curve). The HS fraction estimate is $92 \%$.

As stated above, all HS fractions are given relative to references measured on $5 \mathrm{ML}$ Fe-pypyr on $\mathrm{Au}(111)$. In the following, we estimate the potential deviation between the relative and absolute HS fractions. In Figure S4, the spectrum used as a HS reference (green curve) in the present manuscript is fitted with the HS and LS references from Ref. 3 using Equation S4. A HS fraction of $92 \%$ is inferred from the fit (black curve in Figure S4). Therefore, the absolute HS fractions are approximately a factor 0.9 lower than the indicated relative values. The main motivations to employ the HS and LS references acquired on $\mathrm{Au}(111)$ are: (i) the spectra were measured under the very same conditions as all other spectra shown in the present study and (ii) fits with the references obtained on $\mathrm{Au}(111)$ were systematically better than with that from Ref. 3 .

\section{S4 Spectra of the Fe-pypyr fragments on $\mathrm{Au}(111)$ for dif- ferent temperatures}

The black and blue curves in Figure S5 are XA spectra acquired on 0.4 ML Fe-pypyr on $\mathrm{Au}(111)$ at $300 \mathrm{~K}$ and $100 \mathrm{~K}$, respectively. These spectra are essentially the same, indicating that the fragmented Fe complex is not affected by a variation of temperature. 


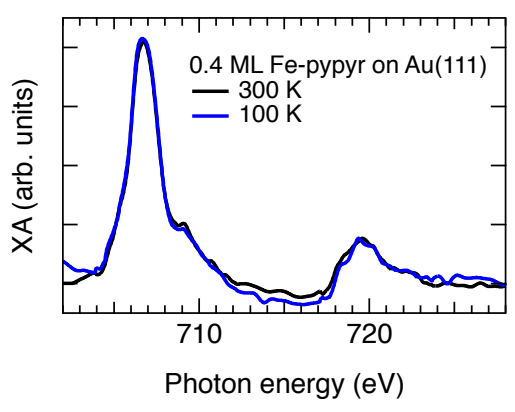

Figure S5: XA spectra acquired on 0.4 ML Fe-pypyr on $\mathrm{Au}(111)$ at $300 \mathrm{~K}$ (black curve) and at $100 \mathrm{~K}$ (blue curve).

\section{S5 Identification of the dissociated molecules}

To obtain further information about the dissociated complexes on $\mathrm{Au}(111)$, scanning tunneling microscopy was carried out at $4 \mathrm{~K}$. Figures S6a,b show typical topographs acquired on $\mathrm{Au}(111)$ upon deposition of Fe-pypyr. Structures with an apparent height of approximately $150 \mathrm{pm}$ and a length of $1.2 \mathrm{~nm}$ (right ellipses in Figure S6a) match in size and symmetry with phenanthroline (phen) ligands (expected length of approximately $0.9 \mathrm{~nm}$ between the outermost hydrogen atoms). Furthermore, this structure is very similar to that of phen ligands on $\mathrm{Co} / \mathrm{Cu}(100)$ (Ref. 4) and on $\mathrm{Au}(111)$ (Ref. 5) and tends to form dimers (left ellipse in Fig. S6a) ${ }^{5}$. We therefore assign these structures to phen ligands, which most probably result from the fragmentation of Fe-pypyr on $\mathrm{Au}(111)$.

Other areas of the same sample exhibit dense structures of adsorbates (Fig. S6b). The size and shape of the adsorbates, with an apparent height of approximately $400 \mathrm{pm}$, are compatible with $\left[\mathrm{Fe}\left(\operatorname{pypyr}\left(\mathrm{CF}_{3}\right)_{2}\right)\right]^{+}$as indicated by the superimposed scaled molecular models. We note that (i) no information is available about the charge state of the fragments and (ii) there may be other fragments on the surface. Indeed, unidentified structures are observed, e.g., the yellowish structures in Figure S6a.

Multiplet calculations were performed with the multiX package ${ }^{6}$ to simulate the XA spectra of the fragments. Figure S6c shows the calculated spectra for an $\mathrm{Fe}^{2+}$ ion in an octahedral crystal field, where the ligands are considered as point charges. The red and blue 
curves are obtained for a crystal-field strength of 0.15 and $0.8 \mathrm{e} / \AA$, respectively (Fig. S6c). These curves are rather similar to the XA spectra of $\mathrm{Fe}^{\mathrm{II}}$ spin-crossover complexes in the HS and LS states, respectively (see for instance Fig. 1c of the main text for comparison). Indeed, the $L_{3}$ edge of the red curve (HS) has a more developed multiplet structure with additional peaks compared to the $L_{3}$ edge of the blue curve (LS). Moreover, the energy of the $L_{3}$ edge of the red curve (HS) is lower than that of the blue curve (LS). For the sake of generality and comparison with other complexes, the multiplet calculations were realized with a limited set of parameters (crystal-field strength and core-hole broadening). More sophisticated calculations would consider the non-perfect octahedral symmetry of the crystal field and scale the spin-orbit coupling and Coulomb interaction.

In the following, we refer to the coordination number $(\mathrm{CN})$ to distinguish between different $\mathrm{Fe}^{\mathrm{II}}$-ligand cages. All the cages were constructed from the $\mathrm{CN} 6$ structure, from which point charges have been removed. Therefore, this approximation assumes no relaxation of $\mathrm{Fe}^{\mathrm{II}}$ cage upon removal of ligands. The XA spectrum of $\left[\mathrm{Fe}\left(\operatorname{pypyr}\left(\mathrm{CF}_{3}\right)_{2}\right)\right]^{+}$, the compound presumably observed in the STM experiments (Fig. S6b), is simulated by considering an $\mathrm{Fe}^{2+}$ ion with only two points charges (CN2; crystal-field strength of $0.8 \mathrm{e} / \AA$ ). The calculated spectrum of the CN2 structure (black curve in Fig. S6c) is similar to that of the fragmented complexes on $\mathrm{Au}(111)$ (black curve in Fig. 1c of the manuscript). In particular, the adsorption edges of the CN2 structure (black curve) have approximately the same energies as that of the CN6 structure with weak crystal field (red curve). This is in agreement with the experimental evidence that the XA spectrum of fragmented molecules may easily be confused with that of intact SCO complexes in the HS state (section S6). Therefore, the combination of STM data and multiplet calculations suggest that the fragmented complex on $\mathrm{Au}(111)$ is $\left[\mathrm{Fe}\left(\operatorname{pypyr}\left(\mathrm{CF}_{3}\right)_{2}\right)\right]^{+}$.

For completeness, we show that simulated spectra of the CN2 structure are essentially independent of the crystal-field strength (compare lower green and black curves in Fig. S6d calculated for crystal-field strength of 0.2 and $0.8 \mathrm{e} / \AA$, respectively). Furthermore, similar 
spectra are obtained for the CN4 structure (upper green and black curves in Fig. S6d). This implies that, from the XA spectra alone, it is challenging if not impossible to retrieve the composition of the fragments.
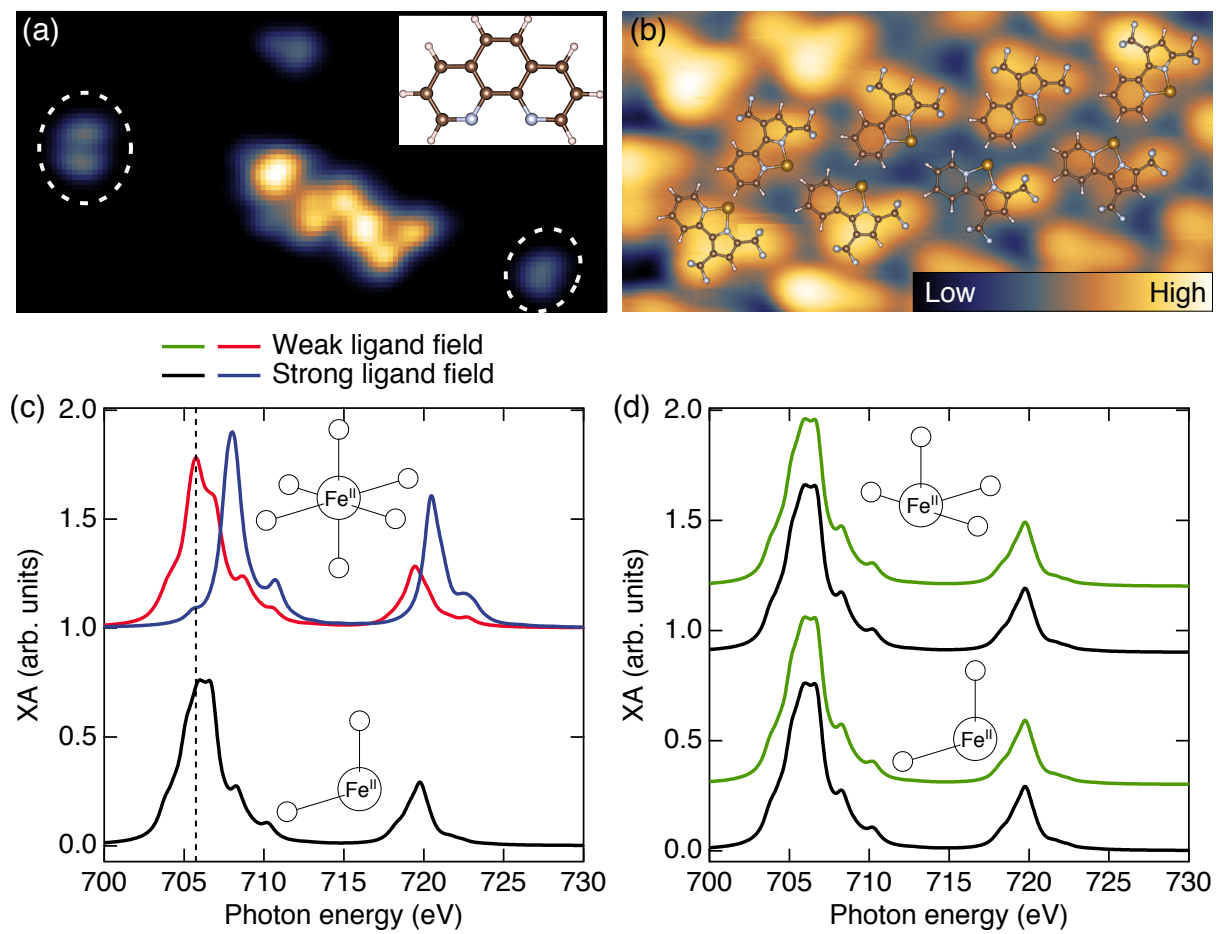

Figure S6: (a,b) Low-temperature STM topographs of Fe-pypyr on Au(111). (a) Isolated phen ligands (dashed ellipses) are observed on the $\mathrm{Au}(111)$ surface along with unidentified structures (yellowish protrusions). (b) Dense structure of molecular adsorbates with superimposed scaled models of $\left[\mathrm{Fe}\left(\operatorname{pypyr}\left(\mathrm{CF}_{3}\right)_{2}\right)\right]^{+}$. The topograph was acquired on the same sample as for (a). Image sizes: (a) $30 \times 20 \mathrm{~nm}^{2}$ and (b) $5 \times 2.5 \mathrm{~nm}^{2}$. (c,d) Multiplet calculations for different Fe-N cages, as depicted in the insets. For every cage, the multiplet calculations were performed for a weak (green and red lines) and for a strong ligand field (black and blue lines). The calculations include a core-hole Lorentzian broadening of $0.5 \mathrm{eV}$ and the energies are shifted by $4.41 \mathrm{eV}$. The scale parameters for the spin-orbit coupling and the Coulomb interaction were left to their default values. 


\section{S6 Distinction between dissociated molecules and com- plexes trapped in a given spin state}

The shape of the Fe $L_{3,2}$ XA spectra of Fe-pypyr on graphene/Ni(111) can, to some extent, be reproduced by linear combination of the reference spectra (red curves in Figs. S7a-b). However, this approach fails to reproduce the correct contribution at some energies, e.g., $706.7 \mathrm{eV}$. In contrast, the fits are drastically improved when a fraction of the molecules are considered dissociated (with spectral features similar to that of dissociated Fe-pypyr/Au(111)) (black curves in Figs. S7a-b). The spectra are fit by a linear combination of (i) the dissociatedcomplex spectrum $\left(X A S_{\text {Diss. }}\right)$ measured on Fe-pypyr/Au(111), (ii) the HS $X A S_{\mathrm{HS}}$ and (iii) the LS $X A S_{\mathrm{LS}}$ references:

(a)
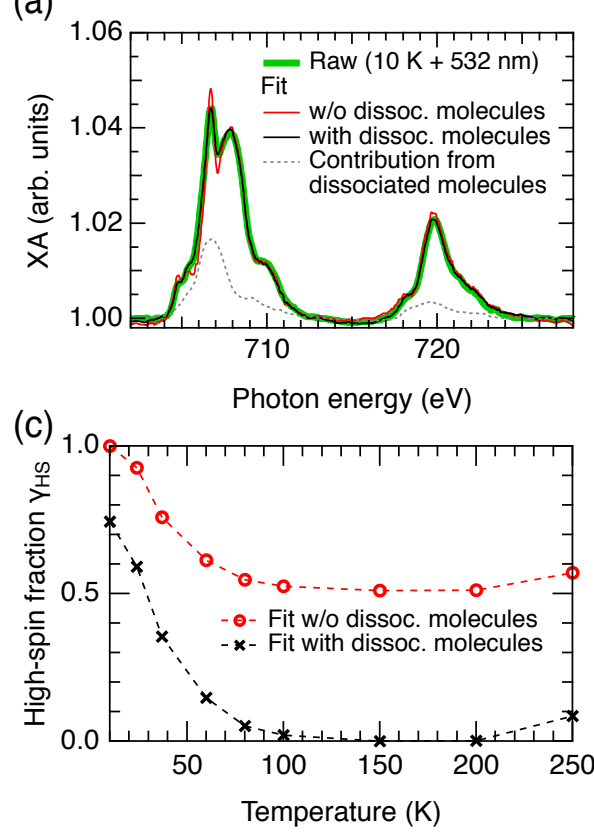

(b)

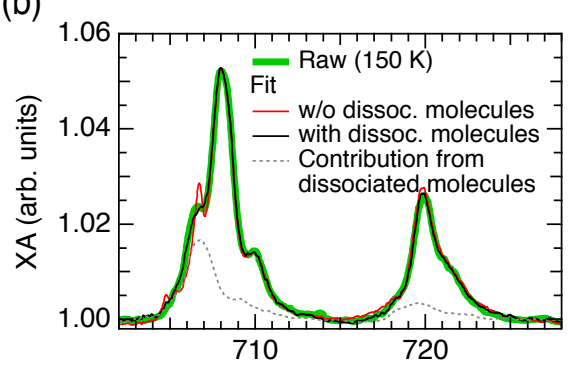

Photon energy (eV)

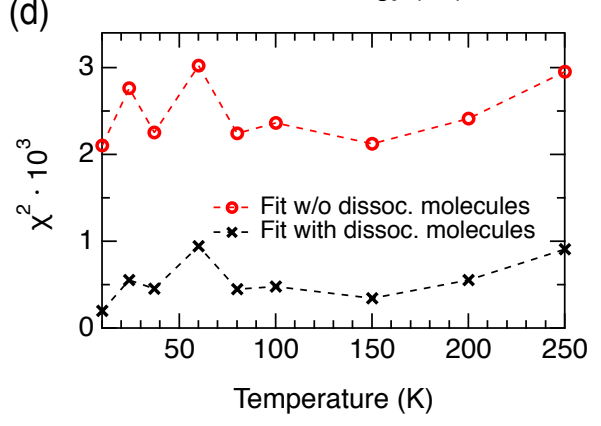

Figure S7: Illustration of the fit quality using Equations S4 (red) and S5 (black) to the spectra acquired at (a) $10 \mathrm{~K}$ under illumination and at (b) $150 \mathrm{~K}$ on $0.4 \mathrm{ML}$ Fepypyr/graphene/Ni(111) (green). (c) Evolution of the HS fraction and (d) the deviation of the data to the fits $\left(\chi^{2}\right)$ as a function of temperature by considering all molecules being intact (Equation S4, red) or by assuming that a fraction of molecules are dissociated (Equation S5, black). 


$$
X A S_{\text {mix }}=a\left\{\gamma_{\text {Diss. }} X A S_{\text {Diss. }}+\left(1-\gamma_{\text {Diss. }}\right)\left[\gamma_{\mathrm{HS}} X A S_{\mathrm{HS}}+\left(1-\gamma_{\mathrm{HS}}\right) X A S_{\mathrm{LS}}\right]\right\}
$$

where $\gamma_{\text {Diss. }}$ and $\gamma_{\mathrm{HS}}$ are the fraction of dissociated molecules and the fraction of HS among the intact complexes, respectively.

On a given sample, $\gamma_{\text {Diss. }}$ is first determined and considered as constant. For this purpose, the spectra acquired at $150 \mathrm{~K}$ are assumed to be in a pure LS state and fit to Equation S5 imposing $\gamma_{\mathrm{HS}}=0 . \gamma_{\text {Diss. }}$ is inferred from the fit and considered constant for the next steps. Then, all spectra, acquired at different temperatures, are fit using Equation S5 with $a$ and $\gamma_{\mathrm{HS}}$ as adjustable parameters.

The complete procedure is illustrated for the sample 0.4 ML Fe-pypyr/graphene/Ni(111). The fit of the spectrum acquired at $150 \mathrm{~K}$ to Equation S5 (imposing $\gamma_{\mathrm{HS}}=0$ ) leads to $\gamma_{\text {Diss. }}=0.30$. All spectra are then fit using Equation S5, where $\gamma_{\text {Diss. }}$ is constrained to 0.30 and $a$ and $\gamma_{\mathrm{HS}}$ are adjustable parameters. The obtained fits reproduce the data well as shown in Figures S7a-b (black curves) for two different temperatures. The evolution of the HS fraction $\left(\gamma_{\mathrm{HS}}\right)$ as a function of temperature is displayed in Figure S7c (black crosses). It is significantly different from the HS fraction inferred from fits to Equation S4, which assumes that all molecules are intact (red circles in Fig. S7c).

To compare the quality of the fits to Equations S4 and S5, the corresponding sums of the squared difference $\left(\chi^{2}\right)$ are calculated. Although both fitting functions have the same number of fit parameters (two in each case), $\chi^{2}$ is significantly smaller when the spectra are fit to Equation S5 (black symbols in Fig. S7d), indicating that Equation S5 is more appropriate to describe the data. 


\section{S7 $0.4 \mathrm{ML}$ Fe-pypyr on $\mathrm{Co} / \mathrm{Cu}(100)$}

The XA spectra at the $\mathrm{Fe} L_{3,2}$ edges on $0.4 \mathrm{ML}$ Fe-pypyr on $\mathrm{Co} / \mathrm{Cu}(100)$ are significantly different from the ones obtained from the HS and LS references, respectively. Instead, they are very similar to that of Fe-pypyr on $\mathrm{Au}(111)$ and are therefore indicative of dissociated complexes (Fig. S8). Furthermore, as these dissociated complexes have a non-vanishing XMCD signal (gray spectrum in Fig. S8), they have magnetic moments and their moments are mostly aligned. The alignment of the magnetic moment is realized by a ferromagnetic coupling between the dissociated complexes and the Co layer, as previously observed on other systems ${ }^{7-10}$.

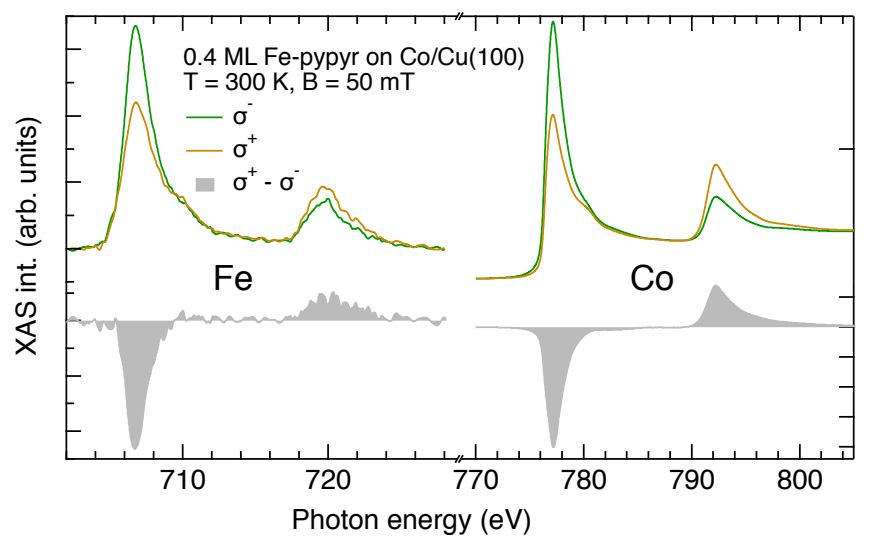

Figure S8: XA spectra of $0.4 \mathrm{ML}$ Fe-pypyr on $\mathrm{Co} / \mathrm{Cu}(100)$ acquired at the Fe and $\mathrm{Co} L_{3,2}$ edges at $300 \mathrm{~K}$ under illumination with circularly polarized left (green) and right (orange) photons. The gray spectrum is the difference (XMCD).

\section{S8 Comparison of dissociated molecules}

XA spectra of dissociated Fe-pypyr complexes on $\mathrm{Au}(111)$ and on $\mathrm{Co} / \mathrm{Cu}(100)$ are identical within the noise level (dashed black, solid blue and solid green curves in Fig. S9). In addition, within the noise level, these spectra are indistinguishable from the contributions of dissociated Fe-pypyr complexes on graphene/Ni(111) (red curve in Fig. S9). This suggests that the fragments are the same on the different surfaces. 


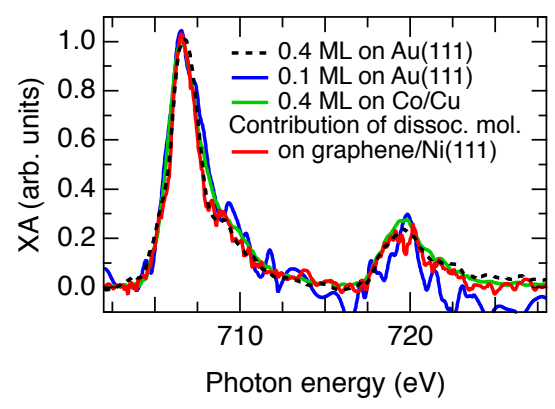

Figure S9: Comparison of the XA spectra acquired on 0.1 ML Fe-pypyr on Au(111) (blue), $0.4 \mathrm{ML}$ Fe-pypyr on $\mathrm{Au}(111)$ (dashed black) and on $\mathrm{Co} / \mathrm{Cu}(100)$ (green) along with the contributions from dissociated molecules on $0.4 \mathrm{ML}$ Fe-pypyr/graphene/Ni(111) (red). The latter contributions are obtained by removing $\left(1-\gamma_{\text {Diss. }}\right) X A S_{L S}$ from the spectrum acquired at $150 \mathrm{~K}$ on graphene $/ \mathrm{Ni}(111)$.

\section{S9 Peculiar results of $0.2 \mathrm{ML}$ Fe-pypyr on $\mathrm{Au}(111)$ pre- pared under inappropriate conditions}

Figure S10a shows the evolution of the XA spectra as a function of temperature on 0.2 ML Fepypyr $/ \mathrm{Au}(111)$. A hasty analysis of these data would lead to the following interpretation: Fe-pypyr molecules in direct contact to $\mathrm{Au}(111)$ exhibit switching while a fraction of the molecules are locked in the HS state (red circles in Fig. S10b). However, in the light of the detailed analysis performed on other samples and because these results are not reproducible, the conclusion should be significantly different.
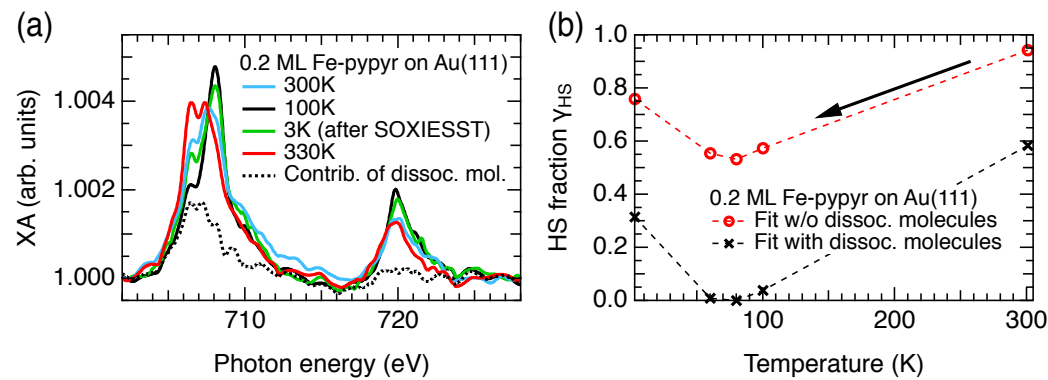

Figure S10: (a) XA spectra of 0.2 ML Fe-pypyr on $\mathrm{Au}(111)$ acquired at different temperatures after the first evaporation process, i.e., after insufficient degassing of the Knudsen cell. (b) Corresponding HS fraction inferred from fits to Equation S4 (red circles) and Equation S5 (black crosses) of the sample at different temperatures. 
First, the presumed fixed fraction of HS molecules actually corresponds to a fraction of dissociated molecules (approximately $30 \%$ ). Second, we prepared several samples with subML coverages of Fe-pypyr on $\mathrm{Au}(111)$. Only one sample exhibited indication of a fraction of switching molecules. It was the first sample of the series prepared after short degassing of the Knudsen cell. We therefore suggest that the switchable molecules are not in direct contact to the $\mathrm{Au}(111)$ but most probably are located on a thin insulating layer of fragments, originating from the degassing of the Knudsen cell. Note that similar results can be obtained when fragments are coevaporated with the complexes. XAS at the Fe edges is insensitive to the eventual Fe-free fragment layer.

\section{References}

(1) Nakajima, R.; Stöhr, J.; Idzerda, Y. U. Electron-Yield Saturation Effects in L-Edge X-Ray Magnetic Circular Dichroism Spectra of Fe, Co, and Ni. Phys. Rev. B 1999, 59, 6421-6429.

(2) Kipgen, L.; Bernien, M.; Ossinger, S.; Nickel, F.; Britton, A. J.; Arruda, L. M.; Naggert, H.; Luo, C.; Lotze, C.; Ryll, H. et al. Evolution of Cooperativity in the Spin Transition of an Iron(II) Complex on a Graphite Surface. Nat. Commun. 2018, 9, 2984.

(3) Rohlf, S.; Gruber, M.; Flöser, B. M.; Grunwald, J.; Jarausch, S.; Diekmann, F.; Kalläne, M.; Jasper-Toennies, T.; Buchholz, A.; Plass, W. et al. Light-Induced Spin Crossover in an Fe(II) Low-Spin Complex Enabled by Surface Adsorption. J. Phy. Chem. Lett. 2018, 9, 1491-1496.

(4) Gueddida, S.; Gruber, M.; Miyamachi, T.; Beaurepaire, E.; Wulfhekel, W.; Alouani, M. Exchange Coupling of Spin-Crossover Molecules to Ferromagnetic Co Islands. J. Phys. Chem. Lett. 2016, 7, 900-904. 
(5) Gopakumar, T. G.; Bernien, M.; Naggert, H.; Matino, F.; Hermanns, C. F.; Bannwarth, A.; Mühlenberend, S.; Krüger, A.; Krüger, D.; Nickel, F. et al. Spin-Crossover Complex on $\mathrm{Au}(111)$ : Structural and Electronic Differences Between Mono- and Multilayers. Chem. Eur. J. 2013, 19, 15702-15709.

(6) Uldry, A.; Vernay, F.; Delley, B. Systematic Computation of Crystal-Field Multiplets for X-Ray Core Spectroscopies. Phys. Rev. B 2012, 85, 125133.

(7) Scheybal, A.; Ramsvik, T.; Bertschinger, R.; Putero, M.; Nolting, F.; Jung, T. A. Induced Magnetic Ordering in a Molecular Monolayer. Chem. Phys. Lett. 2005, 411, $214-220$.

(8) Wende, H.; Bernien, M.; Luo, J.; Sorg, C.; Ponpandian, N.; Kurde, J.; Miguel, J.; Piantek, M.; Xu, X.; Eckhold, P. et al. Substrate-Induced Magnetic Ordering and Switching of Iron Porphyrin Molecules. Nat. Mater. 2007, 6, 516-520.

(9) Candini, A.; Bellini, V.; Klar, D.; Corradini, V.; Biagi, R.; De Renzi, V.; Kummer, K.; Brookes, N. B.; del Pennino, U.; Wende, H. et al. Ferromagnetic Exchange Coupling between Fe Phthalocyanine and Ni(111) Surface Mediated by the Extended States of Graphene. J. Phys. Chem. C 2014, 118, 17670-17676.

(10) Gruber, M.; Ibrahim, F.; Boukari, S.; Isshiki, H.; Joly, L.; Peter, M.; Studniarek, M.; Da Costa, V.; Jabbar, H.; Davesne, V. et al. Exchange Bias and Room-Temperature Magnetic Order in Molecular Layers. Nat. Mater. 2015, 14, 981-984. 